\section{Vaporization of Lactic Acid as an Aerial Bactericide}

Is an earlier communication ${ }^{1}$, an account was given of the bactericidal action of lactic acid vapour. Further work has shown that the methods of vaporization used until that time were inefficient, and that effective bactericidal action is obtained when the concentration of acid vapour in the air is $3.5 \mathrm{mgm}$./ cu. metre.

Lactic acid cannot be boiled at ordinary pressures without decomposition. Dropping the acid or its aqueous solution on to a hot plate also leads to considerable loss of acid. Dispersal as a fine spray is satisfactory from the point of view of avoiding loss or decomposition of acid; but for continuous operation needs cumbersome and expensive apparatus. Lactic acid is, however, readily volatile in superheated steam, and two forms of apparatus for effecting this have been devised and are shown in the accompanying diagram. With appropriate modifications they are, of course, also suitable for the vaporization of other bactericidal substances volatile in steam.
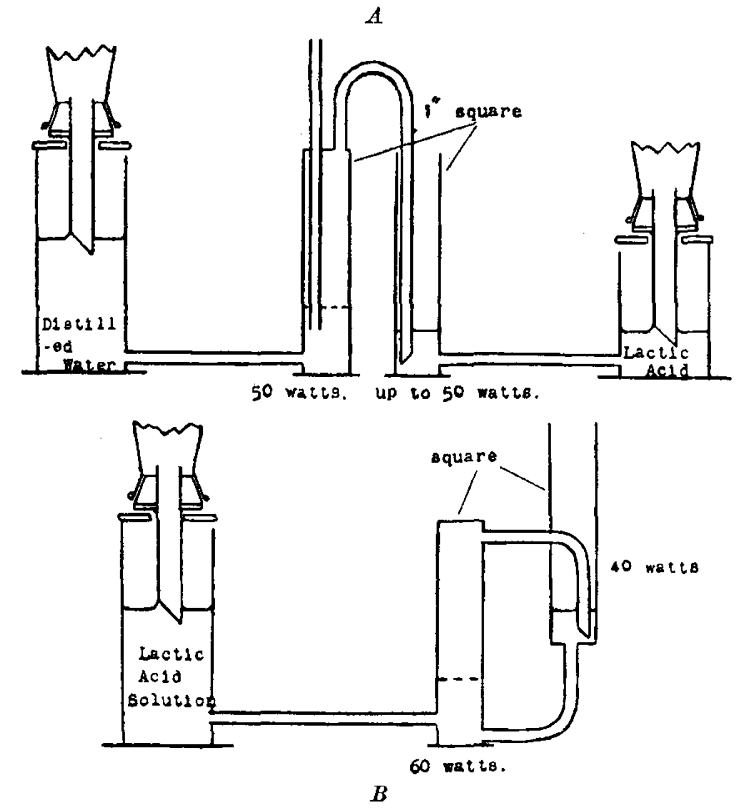

In type $A$, water is fed from a constant-head feed system into a closed electrically heated boiler. The steam from this boiler is then allowed to bubble through the lactic acid in an electrically heated pot into which the lactic acid is fed from another constant-head feed system. The rate of evolution of lactic acid vapour depends on $(a)$ the rate of steam flow, to which it is directly proportional, (b) the temperature of the pot, which is maintained at any desired point between $120^{\circ}$ and $180^{\circ} \mathrm{C}$. by adjusting the heat supply.

In type $B$ an aqueous solution of lactic acid is fed into a closed electrically heated boiler from a single constant-head feed system. The water is largely boiled off and the concentrated lactic acid passes into an electrically heated pot. The steam from the first boiler is allowed to blow off through the lactic acid in this pot. The wattages of the two heaters are adjusted to maintain the second pot between $120^{\circ}$ and $180^{\circ} \mathrm{C}$. For given wattages the temperature of the second pot and the output of vaporized lactic acid are controlled by the concentration of the initial lactic acid solution, which may be varied from zero up to 20 per cent lactic acid by weight. For smooth working the boiler must have adequate thermal capacity; a mass of $250 \mathrm{gm}$. of brass is usually sufficient, but twice this is preferable.

Both types, as illustrated, have a maximum output of about $12 \mathrm{gm}$. of lactic acid vaporized per hour, and have been constructed mainly from brass, with all joints brazed. Corrosion of the metal and formation of non-volatile polymers appear to be negligible under working conditions ; but it is probably desirable to flush out the system occasionally. Distilled water must, of course, always be used to avoid furring up the boiler.

\section{J. E. Lovelock.}

O. M. LIDWELL.

W. F. RAYMOND.

National Institute for Medical Research, London, N.W.3. May 16.

${ }^{1}$ Lovelock, J. E., Lidwell, o. M., and Raymond, W. F., NATURE, 153, 20 (1944).

\section{Role of Manganese in the Biological Synthesis of Ascorbic Acid}

I HAVE adduced evidence ${ }^{1}$ that manganese has a specific role in the synthesis of ascorbic acid by plants and animals. Until now the evidence of the synthesis of ascorbic acid in presence of manganese has been chemical; that is, by titration with the indophenol reagent. Evidence that the indophenolreducing substance synthesized is identical with vitamin $\mathrm{C}$ has now been obtained.

Phaseolus radiatus seeds were germinated in distilled water and in separate and mixed dilute solutions of sodium chloride, magnesium sulphate and manganous chloride. The grains germinated in solutions containing manganese, within certain minimal concentration, alone showed a largely increased synthesis of ascorbic acid; the other salts had no effect upon the synthesis of ascorbic acid. Young growing guinea pigs were divided into three groups and kept on a scorbutic diet of oatmeal, 63 ; bran, 10 ; casein, 20 ; linseed oil, 2 ; codliver oil, 2 ; sodium chloride, 1 ; calcium phosphate, 2. One group was supplemented daily with $0.5 \mathrm{mgm}$. of synthetic ascorbic acid. The second group was supplemented with a given amount of Phaseolus radiatus germinated in distilled water for one day and calculated to contain $0.5 \mathrm{mgm}$. of total (reduced and dehydro-) ascorbic acid. The third group was similarly supplemented with a given (smaller) weight of Phaseolus radiatus germinated in 0.002 per cent manganese solution for one day and containing $0.5 \mathrm{mgm}$. of total ascorbic acid. The experiment lasted three weeks.

The results are given in the accompanying table. The two groups supplemented with germinated Phaseolus radiatus showed exact parallelism in their growth-rate, and closely agreed with the growth-rate of the group supplemented with synthetic ascorbic acid. This establishes the identity of the increased indophenol-reducing substance synthesized by Phaseolus radiatus when germinated in 0.002 per cent manganese solution with vitamin $\mathrm{C}$.

In another set of experiments guinea pigs (120$150 \mathrm{gm}$. in weight) kept on the above scorbutic diet were divided into two groups. One group was 